\title{
18
}

\section{Placement, Grounding, and Mental Content}

\author{
Kelly Trogdon
}

\section{Introduction}

One central issue concerning philosophical methodology is this: what concepts should go into our philosophical toolbox? That is to say, what notions are appropriate to rely on in doing philosophy? This issue is relevant not only to how we should go about addressing philosophical problems but also how we're to formulate those problems in the first place. There is a burgeoning literature on the notion of grounding. I'm a proponent of grounding - I think the notion of grounding is coherent and theoretically useful. ${ }^{1}$ Supposing that the notion of grounding belongs in our philosophical toolbox, what consequences might this have for familiar philosophical problems? In this chapter I focus on what Jackson (1998) calls placement problems - problems concerning how the manifest facts (e.g. facts concerning ordinary macroscopic objects, the mental, and the moral) "fit into" the world given that the world is ultimately physical in nature. If we formulate placement problems in terms of grounding, we should expect new possibilities to open up with respect to how to solve them. My goal

\footnotetext{
${ }^{1}$ See Audi 2012, Fine 2012, Raven 2012, Rosen 2010, and Schaffer 2009 for defenses of the coherence and theoretical utility of the notion. For introductions to the notion of grounding and reviews of recent work on the notion, see Bliss and Trogdon 2014, Clark and Liggins 2012, Correia and Schnieder 2012, Raven forthcoming, and Trogdon 2013a.
} 
in this chapter is to show that this is precisely what happens with respect to the content placement problem, the problem of how to fit facts concerning mental content into the actual world given that it's ultimately physical in nature.

\section{Placement: modal entailment vs. grounding}

We begin with the notion of a placement problem. A familiar take on what it is to address such a problem - what I'll call the modal view of placement - has two components. First, to show how a manifest fact fits into the actual world given that it's ultimately physical in nature is to identify broadly physical facts that modally entail the manifest fact, where the modality in question is metaphysical in nature. (We could put the claim in terms of supervenience as well.) Second, the broadly physical facts include facts concerning the physicalnomic profiles of physical properties, events, and objects. To specify the physical-nomic profile of an entity is to specify facts concerning the nomic relations that entity bears to other physical entities as well as facts concerning the nomic relations that physical entities bear to it. Call these the metaphysical and nomic takes on the fitting-into relation and broadly physical truths, respectively. So the modal view of placement gives pride of place to modality twice over: the fitting-into relation is understood in purely modal terms, and the domain of facts with which we wish to relate the manifest facts include certain modal facts.

Placement problems and the modal view of placement have played an important role in philosophy for the past thirty years or so. Think, for example, about contemporary metaphysics of mind. One placement problem with respect 
to the mental that has received an enormous amount of attention is this: given that the actual world is ultimately physical in nature, how do the phenomenal facts - the facts about which phenomenal properties we instantiate - fit into the world? The non-reductive physicalist thinks she has a solution to the phenomenal placement problem, and a familiar formulation of her thesis is this: while phenomenal and physical properties are distinct, facts concerning the physical-nomic profiles of instantiated physical properties modally entail the phenomenal facts.

An alternative to the metaphysical take on the fitting-into relation is the following: to show how a manifest fact fits into the actual world given that it's ultimately physical in nature is to identify broadly physical facts that ground the manifest fact in question. What reason do we have to take the grounding take on the fitting-into relation seriously? Well, it's natural to think that part of what it is for a manifest fact to fit into the world is for there to be an explanation of that manifest fact in terms of broadly physical facts. And proponents of grounding claim that grounding and explanation are connected in a way that modal entailment and explanation aren't. More specifically, the idea is that grounding is either an explanatory relation (Dasgupta 2014, Fine 2012, and Litland 2013) or a non-explanatory, explanation-backing relation (Audi 2012 and Schaffer 2012), while modal entailment is neither sort of relation.

My interest here, however, isn't the grounding take on the fitting-into relation per se. Instead, I'm interested in the picture of placement that emerges if we combine the grounding take on the fitting-into relation with a grounding 
take on broadly physical facts. The conception of broadly physical facts I have in mind is this: the broadly physical facts include not only facts concerning the physical-nomic profiles of physical entities but their physical-grounding profiles as well. To specify the physical-grounding profile of an entity is to specify what physical facts ground the facts about that entity, as well as what physical facts are grounded by the facts about that entity. While the modal view of placement consists of the metaphysical take on the fitting-into relation and the nomic take on broadly physical facts, let the grounding view of placement be the conjunction of the grounding takes on these notions set out above. Just as modality figures in the modal view of placement twice over, grounding figures in the grounding view of placement twice over: the fitting-into relation is understood in terms of grounding, and the domain of facts with which we wish to relate the manifest facts include certain facts about what grounds what.

If we formulate placement problems in terms of grounding along the lines described above we should expect new possibilities to open up with respect to how to solve them. I'm going to argue that this is so with respect to the following placement problem in particular: given that the actual world is ultimately physical in nature, how do the content facts - the facts about what our concepts refer to - fit into the world?

Our point of departure is the informational approach to the content of concepts championed by Fodor (1990), Dretske (1995, Ch. 1), and others. This approach is naturally viewed as one way of (partially) answering the content placement problem, where either the modal view of placement or a combination 
of the modal and grounding views - one that embraces the grounding take on the fitting-into relation and the nomic take on broadly physical facts - is operating in the background. On the former view of placement the informational approach has it that certain broadly physical facts - facts concerning the physical-nomic profiles of physical entities in particular modally entail the content facts, while on the latter view of placement this approach to content has it that these broadly physical facts ground the content facts.

The informational approach to content understood in either way, as well as the program of naturalizing content in general, has fallen out of favor. Many (most?) philosophers interested in mental representation are skeptical that anything like the informational approach is even close to being right. But suppose that the grounding view of placement is correct rather than the modal view or the combination of these views described above. In this case, a new sort of informational approach emerges: certain broadly physical facts concerning the physical-nomic and physical-grounding profiles of physical entities ground the content facts. What might such an account look like? In this chapter I propose a theory of content along these lines, one that's modeled on Fodor's (1990) asymmetric dependence theory but has important advantages over it. The literature on the asymmetric dependence theory of content and naturalistic theories of content more generally ran dry a while ago, but with recourse to grounding it will be profitable to consider them afresh. 


\section{Fodor's asymmetric dependence theory}

While you're probably familiar with Fodor's theory, it's important that we get the details of the asymmetric dependence theory right since the theory that I go on to offer is modeled on his theory. (So be patient!) Let's focus on the concept RABBIT. Fodor argues that informational theories that proceed upon a distinction between normal and abnormal circumstances in which RABBIT is activated face insurmountable problems, so he proposes an entirely different type of informational theory. Consider the set $\Delta$, where $\Delta$ consists of all and only the nomic relations that have some property or other in their first argument place and RABBIT in their second. Although he doesn't put things in quite this way, as I read Fodor his key idea is this: if there is a relation of the appropriate sort that induces an ordering with respect to the members of $\Delta$ such that rabbit/RABBIT (the nomic relation between being a rabbit and RABBIT) occupies a special position, it follows that being a rabbit is the content of RABBIT. Call this type of informational theory the nomic ordering theory. Fodor urges that we abandon informational theories that appeal to a distinction between normal and abnormal conditions and focus on developing nomic ordering theories instead.

Fodor develops a particular nomic ordering theory, the asymmetric dependence theory. Any nomic ordering theory has two related parts. Continuing with our focus on RABBIT, the first concerns just what the relevant ordering is of the members of $\Delta$. Fodor works with the following: there is a particular relation, $\mathrm{R}$, such that rabbit/RABBIT bears $\mathrm{R}$ to every other member of $\Delta$, while none of them bear $\mathrm{R}$ to rabbit/RABBIT. The second concerns just what 
relation induces the relevant ordering. Let's say that, for any properties $\mathrm{P}$ and $\mathrm{Q}$, if there is a nomic relation between $\mathrm{P}$ and RABBIT and a nomic relation between Q and RABBIT, then the former counterfactually determines (cdetermines for short) the latter just in case, ceteris paribus, were $\mathrm{P}$ not nomically related to RABBIT, $\mathrm{Q}$ wouldn't be either. Fodor works with the idea that $\mathrm{R}$ is the relation of $\mathrm{c}$-dependence. Putting all of this together, the asymmetric dependence theory (with respect to RABBIT) is this: it's necessary that (i) if rabbit/RABBIT c-determines every other member of $\Delta$, and (ii) no member of $\Delta$ distinct from rabbit/RABBIT c-determines rabbit/RABBIT, then being a rabbit is the content of RABBIT.

A couple of points worth keeping in mind about the asymmetric dependence theory are as follows. First, while the theory is referentialist in nature (i.e. it identifies the content of a concept with its referent), it's compatible with the denial of Fodor's radical nativism about concepts. Second, the theory aims to provide a sufficient condition for a property to be the content of a concept. So to point out that the asymmetric dependence theory apparently doesn't cover empty concepts, logical concepts, concepts whose referents are response-dependent properties, and so on isn't necessarily to undermine the theory. Were the aim instead to provide a necessary condition for something to be the content of a concept, we would have a problem here. (The same considerations apply to the theory of content I set out later.)

On the modal view of placement the asymmetric dependence theory as stated is a candidate solution to the content placement problem, as (i) and (ii) 
from above are broadly physical propositions. (So we can speak of broadly physical propositions in addition to broadly physical facts - for the purposes of this chapter I understand facts to be true propositions.) On a view of placement that mixes the grounding take on the fitting-into relation and the nomic take on broadly physical truths, the following variation of the asymmetric dependence theory is a candidate solution to the content placement problem: it's necessary that if (i) and (ii) from above are true, then the truth of (i) and (ii) grounds the fact that being a rabbit is the content of RABBIT. (This theory is also a candidate solution to the problem given the grounding take of placement as well.)

Given that there is a nomic relation between being a hare and RABBIT, the sufficient condition the asymmetric dependence theory identifies for being $a$ rabbit to be the content of RABBIT is satisfied only if rabbit/RABBIT c-determines hare/RABBIT and not vice versa. In other words, the following qualified counterfactual is true: ceteris paribus, were being a rabbit not nomically related to RABBIT, being a hare wouldn't be either, but not vice versa. Suppose that we establish that this qualified counterfactual is true only if a particular nonqualified counterfactual is true as well. (Candidate example: were it not the case that hares cause the activation of RABBIT it still would be the case that rabbits cause the activation of RABBIT.) It's important to note that Fodor doesn't see his job as requiring that he provide arguments for either the qualified or nonqualified counterfactuals just mentioned, and the same goes for the other counterfactual commitments of his theory. What he's trying to do is address “...Brentano's problem [i.e. the problem of content placement] by showing that 
there are naturalistically specifiable, and atomistic sufficient conditions for a physical state to have an intentional content," and "In that context, I get to stipulate the counterfactuals" $(1990,96) .^{2}$

\section{The problem of Quinean alternatives}

The problem of Quinean alternatives shows that the sufficient condition identified by the asymmetric dependence theory isn't satisfied, as Fodor himself later admits (1994, Ch. 3). Indeed, on the basis of this problem Fodor concludes that no purely informational theory can solve the content placement problem. What is the problem exactly? While you're probably familiar with the problem at least in broad outline, the details again are important, for the theory that I go on to offer doesn't face this problem, or so I argue. (So again, be patient!)

Consider the following property: being some non-detached rabbit proper part or other (being non-detached for short). Not only is there a nomic

\footnotetext{
${ }^{2}$ What sorts of properties distinct from being a rabbit in addition to being a hare are nomically related to RABBIT? The following are plausible candidates: other properties involved in misperception (e.g. being a possum on a dark night), properties involved in non-psychological interventions with respect to RABBIT (e.g. being bumped - the property of having your head bumped in thus-and-so way), properties associated with causal intermediaries between the activation of RABBIT and its distal causes (e.g. properties concerning irradiation patterns on the retina), properties in virtue of which the aforementioned properties are instantiated, and disjunctive properties whose disjuncts include any of the aforementioned properties. Supposing that these properties are nomically related to RABBIT, the sufficient condition identified by the asymmetric dependence theory for being a rabbit to be the content of RABBIT is satisfied only if certain counterfactuals concerning them are true, such as rabbit/RABBIT c-determines bumped/RABBIT.
} 
relation between being a rabbit and RABBIT, but there's a nomic relation between being non-detached and RABBIT as well. ${ }^{3}$ Indeed, it seems that rabbit/RABBIT and non-detached/RABBIT are intensionally equivalent in the following sense: any possible world in which there is a nomic relation between being a rabbit and RABBIT is a world in which there is a nomic relation between being non-detached and RABBIT and vice versa. Let's suppose that they are indeed intensionally equivalent in this sense.

While Fodor doesn't put things in quite this way, the problem arises when we consider the fact that c-determination is intensional in nature. A consequence of the intensional nature of the relation is this: for any properties $\mathrm{P}$ and Q, if (i) there is a nomic relation between P and RABBIT, (ii) there is a nomic relation between $\mathrm{Q}$ and RABBIT, and (iii) P/RABBIT c-determines Q/RABBIT, then (iv) any nomic relation that's intensionally equivalent with

\footnotetext{
${ }^{3}$ You might claim that, while it may be that being non-detached is nomically related to certain concepts, it isn't nomically related to RABBIT. As Gates (1996) points out, however, the method of differences - the test Fodor appeals to in arguing that certain properties (e.g. being a small rabbit) aren't nomically related to RABBIT doesn't suggest that being non-detached isn't nomically related to RABBIT. Indeed, the test suggests that it is. You might also claim that being non-detached isn't nomically related to RABBIT because it doesn't enter into any nomic relations whatsoever. Perhaps the idea is that the property is disjunctive, and disjunctive properties don't enter into nomic relations. Fodor, however, grants that disjunctive properties do stand in nomic relations so long as they aren't open disjunctions. There would be no disjunction problem to begin with if disjunctive properties didn't stand in nomic relations! Moreover, it's unclear that being non-detached is disjunctive in the first place - it strikes me as second-order monadic property and as such is projectible (see Fodor 1997 for relevant discussion).
} 
P/RABBIT c-determines Q/RABBIT, and (v) any nomic relation that's intensionally equivalent with Q/RABBIT is c-determined by P/RABBIT. Given (iv), if rabbit/RABBIT c-determines non-detached/RABBIT then nondetached/RABBIT does as well. And, given (v), if non-detached/RABBIT cdetermines non-detached/RABBIT then it c-determines rabbit/RABBIT as well. Hence, if rabbit/RABBIT c-determines non-detached/RABBIT then nondetached/RABBIT c-determines rabbit/RABBIT. So the very formal features of cdetermination - in particular the fact that it's intensional in nature - ensure that the sufficient condition identified by the asymmetric dependence theory for being a rabbit to be the content of RABBIT is never satisfied. ${ }^{4}$

${ }^{4}$ See Adams and Aizawa 2010 for related discussion. Notice that we get the same result even if we grant that there are possible worlds in which there is a nomic relation between being a rabbit and RABBIT but not being non-detached and RABBIT and there are possible worlds in which there is a nomic relation between being nondetached and RABBIT but not being a rabbit and RABBIT. (It's hard to imagine what such worlds are like, but let's put this matter to the side.) Supposing that there are such worlds, they're radically different from the actual world. As such, they aren't relevant to establishing whether rabbit/RABBIT c-determines non-detached/RABBIT and vice versa. So let's restrict our attention to worlds that are potentially relevant to evaluating these claims - call such world rabbit worlds. Rabbit/RABBIT and nondetached/RABBIT are intensionally equivalent in rabbit worlds - any rabbit world in which there is a nomic relation between being a rabbit and RABBIT is a world in which there is a nomic relation between being non-detached and RABBIT and vice versa. It follows from the intensional nature of c-determination that, for any properties $\mathrm{P}$ and $\mathrm{Q}$, if (i) there is a nomic relation between $\mathrm{P}$ and RABBIT, (ii) there is a nomic relation between $\mathrm{Q}$ and RABBIT, and (iii) P/RABBIT c-determines Q/RABBIT, then (iv) any nomic relation that's intensionally equivalent with P/ RABBIT in rabbit 
Adams and Aizawa (2010) catalogue various objections to the asymmetric dependence theory, as do Rupert (2008) and Ryder (2009). Many objections to the theory proceed upon fairly specific assumptions about how to evaluate counterfactuals (e.g. assumptions regarding the relative proximity of possible worlds to the actual world). These assumptions are contentious, so I don't put a lot of stock in these sorts of objections, and Fodor probably doesn't either. Notice that the problem of Quinean alternatives, however, doesn't seem to proceed upon any contentious assumptions.

\section{The grounding theory}

Recall that the asymmetric dependence theory, as a nomic ordering theory, has two related parts - the first concerns the ordering of the members of $\Delta$, and the second concerns the relation that induces this ordering. The problem of Quinean alternatives shows that, if the members of $\Delta$ are ordered in the way that Fodor claims they are, it can't be the relation of c-determination that induces that ordering. $\mathrm{R}$ isn't the relation of c-determination.

If the asymmetric dependence theory is to be rejected, what might another nomic ordering theory look like? Let's keep the first part of the asymmetric dependence theory intact: continuing with our focus on RABBIT, we'll work with the following: rabbit/RABBIT bears R to every other member of $\Delta$, while none of them bear $\mathrm{R}$ to rabbit/RABBIT. What we're going to change is the second part of theory. The grounding approach to placement suggests that

worlds c-determines $\mathrm{Q} / \mathrm{RABBIT}$, and (v) any nomic relation that's intensionally equivalent with $\mathrm{Q} / \mathrm{RABBIT}$ in rabbit worlds is c-determined by $\mathrm{P} / \mathrm{RABBIT}$. 
we have another option here: $\mathrm{R}$, rather than the relation of c-determination, is a relation defined in terms of grounding. Grounding is a relation between facts (true propositions), and nomic relations aren't facts. So let's say that, for any properties $\mathrm{P}$ and $\mathrm{Q}, \mathrm{P} / \mathrm{RABBIT}$ grounds* $\mathrm{Q} / \mathrm{RABBIT}$ just in case the fact that there is a nomic relation between $\mathrm{P}$ and RABBIT grounds the fact that there is a nomic relation between $\mathrm{Q}$ and RABBIT. The idea is that $\mathrm{R}$ is grounding*. Putting all of this together, the grounding theory (with respect to RABBIT) is this: it's necessary that if (i) rabbit/RABBIT grounds* every other member of $\Delta$, and (ii) no other member of $\Delta$ grounds* rabbit/RABBIT, then the truth of (i) and (ii) grounds the fact that being a rabbit is the content of RABBIT. ${ }^{5}$ On the grounding view of placement, the grounding theory is a candidate solution to the content placement problem, as (i) and (ii) from above are broadly physical propositions .

Before returning to the problem of Quinean alternatives, five points of clarification about how I'm conceiving of the grounding theory are as follows. First, given that there is a nomic relation between being a hare and RABBIT, the sufficient condition identified by the grounding theory for being a rabbit to be the content of RABBIT is satisfied only if rabbit/RABBIT grounds* hare/RABBIT. But when we claim that rabbit/RABBIT grounds* hare/RABBIT, what exactly are we claiming? Well, if there is a nomic relation between being a hare and RABBIT then something sustains the link between these items - there is a

\footnotetext{
${ }^{5}$ If grounding is asymmetric, then, if rabbit/RABBIT grounds* every other member of $\Delta$ it automatically follows that none of these relations grounds* rabbit/RABBIT. But, as the asymmetry of grounding is a matter of controversy (see Rodriguez-Pereyra forthcoming), I'll stay neutral on whether grounding is indeed asymmetric.
} 
"sustaining mechanism" for the link. Laurence and Margolis, for example, write, "A sustaining mechanism is a mechanism in virtue of which a concept stands in the mind-world relation that a causal theory of content like Fodor's takes to be constitutive of content" $(2002,37)$. When we say that rabbit/RABBIT grounds* hare/RABBIT, what we're saying is that the link between being a rabbit and RABBIT is a sustaining mechanism for the link between being a hare and RABBIT.

Second, the claim that the link between being a rabbit and RABBIT sustains the link between being a hare and RABBIT is compatible with the latter having other sustaining mechanisms. So accepting this claim doesn't commit us to denying more familiar stories about what grounds the fact that there is a nomic relation between being a hare and RABBIT. We can consistently maintain, for example, that certain cognitive dispositions are sustaining mechanisms for the link between being a hare and RABBIT as well as the link between being a rabbit and this concept (Aydede and Güzeldere 2005; Bridges 2006; Fodor 1998, Ch. 4; Laurence and Margolis 2002).

Third, to claim that the link between being a rabbit and RABBIT sustains the link between being a hare and RABBIT isn't to deny that the former also cdetermines the latter. So if the sufficient condition identified by the asymmetric dependence theory for being a rabbit to be the content of RABBIT is satisfied only if a particular counterfactual is true and you find this counterfactual independently plausible, claiming that the corresponding condition identified by the grounding theory is also satisfied doesn't require that you reject this 
counterfactual, at least in the absence of further argument. Consider again the following qualified counterfactual: ceteris paribus, were it not the case that there is a nomic relation between being a rabbit and RABBIT there wouldn't be a nomic relation between being a hare and RABBIT either. Suppose that the link between being a rabbit and RABBIT sustains the link between being a hare and RABBIT. If the nearest possible worlds to the actual world in which there isn't a nomic relation between being a rabbit and RABBIT are worlds in which there is no other sustaining mechanism for a link between being a hare and RABBIT, this counterfactual is true. ${ }^{6}$

Fourth, grounding* is defined in terms of partial grounding. It seems that grounding carries modal entailment in the following sense: full grounds modally entail what they ground (Trogdon 2013b). If one fact merely partially grounds another (contingent) fact, however, we don't get this modal consequence. So consider again the claim that rabbit/RABBIT grounds* hare/RABBIT. Were this a claim about full grounding, we would get the implausible consequence that any world in which there is a nomic relation between being a rabbit and RABBIT is a world in which there is a nomic relation between being a hare and RABBIT. If instead the claim concerns mere partial grounding - the intended reading - we don't get this consequence. ${ }^{7}$

\footnotetext{
${ }^{6}$ If there are possible worlds in which there is a nomic relation between being a hare and RABBIT but this fact lacks grounds, such worlds are radically different from the actual world and thus aren't relevant to the evaluation of this counterfactual.

${ }^{7}$ Example of full vs. merely partial grounding: the plurality consisting of the fact that $p$ and the fact that $q$ fully grounds the fact that $\mathrm{p}$ and $\mathrm{q}$, while the fact that $\mathrm{p}$ merely
} 
Fifth, just as Fodor doesn't see his job as requiring that he provide arguments for the counterfactuals the asymmetric dependence theory brings in its train, I don't see my job as requiring that I provide arguments for the grounding claims that the grounding theory brings in its train. What I want to do is show that, bracketing for now the project of providing detailed arguments for the particular counterfactual/grounding commitments of these theories (e.g. rabbit/RABBIT c-determines hare/RABBIT but not vice versa and rabbit/RABBIT grounds* hare/RABBIT), the grounding theory is a more promising nomic ordering theory than the asymmetric dependence theory. ${ }^{8}$

The idea, as I've already indicated, turns on the problem of Quinean alternatives. As we've seen, the intensional nature of c-determination ensures that non-detached/RABBIT c-determines rabbit/RABBIT provided that rabbit/RABBIT c-determines non-detached/RABBIT. This is why c-determination can't be $\mathrm{R}$, the relation that induces the relevant ordering with respect to the

partially grounds the fact that $\mathrm{p}$ and $\mathrm{q}$ (assuming that the fact that $\mathrm{p}$ and the fact that $\mathrm{q}$ are different facts). Returning to the grounding theory, just what we would need to add to the mere partial ground described above to get a full ground is an issue I won't pursue here. It would presumably have to be something about being a hare.

${ }^{8}$ In note 2 I describe various candidates for properties distinct from being a rabbit in addition to being a hare that are nomically related to RABBIT. If these properties are nomically related to RABBIT, then, while the sufficient condition identified by the asymmetric dependence theory for being a rabbit to be the content of RABBIT is satisfied only if certain counterfactuals concerning these properties are true, the corresponding condition identified by the grounding theory is satisfied only if certain grounding claims concerning them are true. Example: rabbit/RABBIT grounds* bumped/RABBIT. 
members of $\Delta$. What we need here is a hyperintensional relation - if $\mathrm{R}$ is hyperintensional, then, for any properties $\mathrm{P}$ and $\mathrm{Q}$, if (i) there is a nomic relation between $\mathrm{P}$ and RABBIT, (ii) there is a nomic relation between $\mathrm{Q}$ and RABBIT, and (iii) P/RABBIT stands in R to Q/RABBIT, it doesn't follow that (iv) any nomic relation that's intensionally equivalent with P/RABBIT stands in $\mathrm{R}$ to Q/RABBIT, and (v) any nomic relation that's intensionally equivalent with $\mathrm{Q} / \mathrm{RABBIT}$ is such that P/RABBIT stands in $\mathrm{R}$ to that relation.

Grounding* is hyperintensional, for grounding itself is hyperintensional. Suppose, for example, that the fact that Socrates exists grounds the fact that $\{$ Socrates\} exists. Socrates and $\{$ Socrates $\}$ are intensionally equivalent, yet the fact that $\{$ Socrates $\}$ exists doesn't ground the fact that $\{$ Socrates $\}$ exists, and the fact that $\left\{\right.$ Socrates exists doesn't ground the fact that Socrates exists. ${ }^{9}$ So it doesn't follow from the nature of grounding* that non-detached/RABBIT grounds* rabbit/RABBIT provided that rabbit/RABBIT grounds* nondetached/RABBIT. Hence, to borrow Fodor's phrasing, we're free to stipulate that, instead of non-detached/RABBIT grounding* rabbit/RABBIT, the latter grounds* the former. Fodor, by contrast, isn't free to stipulate that it's not the case that non-detached/RABBIT c-determines rabbit/RABBIT, given that he's committed to the claim that rabbit/RABBIT c-determines non-detached/RABBIT. So I conclude that, again setting to the side for now the project of providing

${ }^{9}$ The fact that grounding is hyperintensional (as well as non-monotonic) shows that the grounding locution isn't (completely) analyzable in terms of purely modal notions such as supervenience or modal entailment. See Trogdon 2013a for related discussion. 
detailed arguments for the particular counterfactual/grounding commitments of these theories - the grounding theory is a more promising nomic ordering theory than the asymmetric dependence theory, as it has the resources to handle Quinean alternatives. Nolan (2014) suggests that there are many areas in philosophy where we run up against limitations that can be lifted by using hyperintensional resources in our theorizing. I think that the problem Fodor faces in developing a nomic ordering theory is a case in point.

\section{Further thoughts}

While I've argued that the grounding theory is superior to the asymmetric dependence theory in so far as the former but not the latter has the resources to handle Quinean alternatives, I should note that I'm open to the idea that some other nomic ordering theory that appeals to grounding is superior to the grounding theory. What might such a theory look like? ${ }^{10}$ Well, notice that there are other ways in which the members of $\Delta$ might be ordered whereby rabbit/RABBIT has a special status. Here is one example: (i) rabbit/RABBIT both stands in $\mathrm{R}$ to some other element of $\Delta$ and is such that no other element of $\Delta$ stands in $\mathrm{R}$ to it, and (ii) for any element of $\Delta$, if it both stands in $\mathrm{R}$ to some other element of $\Delta$ and is such that no other element of $\Delta$ stands in $\mathrm{R}$ to it, then that relation is rabbit/RABBIT. Suppose that R so understood is grounding*.

Then the idea is that, while some members of $\Delta$ distinct from rabbit/RABBIT

${ }^{10}$ There are hyperintensional relations other than grounding that are R-candidates, such as relations defined in terms of Fine's (1994) notion of real definition. Just what a nomic ordering theory cast in terms of such relations would look like, however, is a matter I won't pursue here. 
might ground* other members of $\Delta$, and some members of $\Delta$ distinct from rabbit/RABBIT might not be grounded* by other members of $\Delta$, rabbit/RABBIT is unique in that it's the only element of $\Delta$ that both grounds some other element of $\Delta$ and itself isn't grounded by any member of $\Delta$. On this proposal, we could accept, for example, that rabbit/RABBIT grounds* hare/RABBIT and maintain that neither rabbit/RABBIT nor any other member of $\Delta$ grounds* bumped/RABBIT (see note 8 ), assuming that the there is no element of $\Delta$ that the latter grounds*. ${ }^{11}$

This proposal is worth further thought, but let's put it to the side and return to the original grounding theory. As we've seen, the sufficient condition identified by the theory for being a rabbit to be the content of RABBIT is satisfied only if certain grounding claims are true. Whether we're justified in believing that these grounding claims are true is of course relevant to whether we should think that the condition identified by the theory is actually satisfied. So I think the next step to take in thinking about the grounding theory is this: articulate plausible diagnostics for grounding - principles that specify the conditions under which claims about what grounds what are plausible - and then see how the grounding claims the theory is committed to fair with respect to these diagnostics.

${ }^{11}$ What about the view according to which $\mathrm{R}$ so understood is c-determination? Well, in this case we don't have to say that rabbit/RABBIT c-determines bumped/RABBIT, which you might think is an improvement over the asymmetric dependence theory. But the new theory still has a problem with Quinean alternatives - the sufficient condition for being a rabbit to be the content of RABBIT in this case isn't satisfied because non-detached/RABBIT c-determines rabbit/RABBIT. 
So what are plausible grounding diagnostics? This is a difficult matter indeed, I think it's one of the most difficult questions proponents of grounding face. The literature on grounding so far has focused on issues such as the formal features and logic of grounding, how the notion of grounding is related to other notions of philosophical interest, and what grounds the facts about what grounds what. These discussions, however, provide little guidance for formulating grounding diagnostics - it's unclear, for example, that anything here could be operationalized into a discovery procedure that doesn't crucially depend on our already having knowledge about what grounds what. (Similar considerations apply to treatments of causation.)

Here are some preliminary remarks about grounding diagnostics - what I say here is quite general, but it at least gets the ball rolling. If our target is a particular grounding claim - this fact grounds that fact - rather than a generic grounding claim - these facts grounds those facts - there are at least three ways we might try to motivate the claim. First, we might appeal to intuitions about the case. Intuitively, the existence and nature of this cup (as I point to a cup), for example, is grounded in the existence and nature of its proper parts. Now, I realize that it's controversial whether intuitions are properly thought of as functioning as evidence for theoretical beliefs. If you're worried about this, read the claim above as conditional in nature: if intuitions serve as evidence for our theoretical beliefs, then one source of evidence for a particular grounding claim is whether we find the claim intuitively plausible. 
Second, we might appeal to grounding principles that entail or suggest that the particular grounding claim is true. There is a plausible general principle concerning parts and wholes that tells us that the existence and nature of this cup is grounded in the existence and nature of its proper parts, or so you might argue. And a grounding principle that's potentially relevant to the grounding theory is the following: if you have reason to believe that one fact grounds another or vice versa, then, if the properties involved in the first fact are more natural than the properties involved in the second fact (in Lewis' 1983 sense), there is reason to believe that the former grounds the latter rather than the other way around. If we have reason to believe that either rabbit/RABBIT grounds* non-detached/RABBIT or vice versa, then this principle recommends that rabbit/RABBIT grounds* non-detached/RABBIT, assuming that being a rabbit is more natural than being non-detached. A natural question to ask of this approach, however, is what makes the grounding principles themselves plausible. $^{12}$

Third - or this may just be a way of answering the question just raised we might consider the particular grounding claim in relation to an explanatory scheme in which it figures. The system of grounding claims itself is appraised on the basis of considerations such as simplicity, breadth, coherence, and non-

\footnotetext{
${ }^{12}$ With regard to the grounding principle mentioned above, if naturalness itself is to be understood ultimately in terms of grounding then it may be that the only plausible diagnostic in the neighborhood here is one for naturalness claims that appeals to grounding. What we want, however, is a diagnostic for grounding claims that appeals to naturalness.
} 
circularity, and the particular grounding claim inherits the plausibility (or lack thereof) of the explanatory scheme itself (Fine 2001). Returning to grounding principles, if we think of grounding principles as either explanatory schemes or aspects of explanatory schemes, here we have a source of evidence for grounding principles as well.

Consider, for example, the claim that the existence and nature of the number 42 is grounded in facts concerning a certain position in an abstract structure. The plausibility of this grounding claim is a function of the overall plausibility of (a particular version of) structuralism about numbers as an explanatory scheme.${ }^{13}$ Returning to the grounding theory, the claim that rabbit/RABBIT grounds* hare/RABBIT, for example, is a part of an explanatory scheme that, if true, (partially) solves the content placement problem. This explanatory scheme is superior to the explanatory scheme embodied by the asymmetric dependence theory, or so I've argued. The relevant theoretical virtue here is that of coherence - the latter explanatory scheme involves counterfactual commitments that are incompatible with the very nature of cdetermination, while the former explanatory scheme doesn't involve grounding commitments that are incompatible with the nature of grounding. Supposing that (i) the explanatory scheme embodied by the grounding theory is superior to any competing explanatory scheme according to which it's not the case that rabbit/RABBIT grounds* hare/RABBIT, and (ii) any competing explanatory scheme that's on a par with the explanatory scheme embodied by the grounding

\footnotetext{
${ }^{13}$ See Shapiro 1997 for a defense of structuralism about mathematical theories.
} 
theory agrees that rabbit/RABBIT grounds* hare/RABBIT (candidate example: the theory I mentioned at the beginning of this section), we thereby have reason to think that this grounding claim is true, or so the idea goes. ${ }^{14}$

The grounding claims that the grounding theory is committed to aren't particularly intuitive, so it seems that, of the three potential routes to motivating the claims described above, the second and third are the most promising. Suppose that one of these grounding claims - let's say the claim that rabbit/RABBIT grounds* hare/RABBIT - not only strikes you as not intuitive but outright false. Here are two things worth considering. First, your impression that the grounding claim is false might result from the fact that you're focusing on what's different about the causal facts - the rabbit and hare parts - rather than what's the same about them - the RABBIT part. For example, if we gloss the question "Does rabbit/RABBIT ground* hare/RABBIT?" as "Do certain facts about what being a rabbit is nomically linked with ground facts about what being a hare is nomically linked with?" you might be inclined to say 'no' in response. But if we gloss the question instead as "Do certain facts about what RABBIT is nomically linked with ground other facts about what RABBIT is nomically linked with?" things are less clear.

Second, there may be holistic considerations that suggest that rabbit/RABBIT grounds* hare/RABBIT, where these considerations carry more evidential weight than the fact that this grounding claim is counterintuitive.

${ }^{14}$ See also Raven 2012 and Schaffer 2009 for preliminary discussions of grounding diagnostics. 
Here's an example illustrating the point. Suppose you're having an experience as of motion. The non-reductive physicalist claims that certain broadly physical facts - say facts concerning recurrent activity between your V1 and MT/V5 neural structures - grounds the fact that you're having an experience as of motion. This particular grounding claim strikes us as false - it strikes us that there is no such connection between these facts. The vast literature on the explanatory gap challenge to physicalism testifies to this fact. The nonreductive physicalist, however, points out that there are powerful holistic considerations in support of an explanatory scheme - the system of grounding claims that non-reductive physicalism embodies - that has this particular grounding claim as a part. One such holistic consideration involves mental causation. Very roughly, the idea is that (i) mental events cause other mental and physical events; (ii) if the mental facts are grounded in certain broadly physical facts we can see how mental causation is possible; hence, (iii) we have reason to think that the mental facts are grounded in certain broadly physical facts (Levine 2001, Ch. 1). Cast in terms of theoretical virtues, the idea is that the explanatory scheme embodied by non-reductive physicalism does well with respect to the virtue of breadth given its implications for mental causation. So, returning to the grounding theory, it may be that the fact that the claim that rabbit/RABBIT grounds* hare/RABBIT is part of a plausible explanatory scheme has more evidential weight than the fact that this grounding claim strikes us as false. 


\section{Conclusion}

I began this chapter with the following idea: if we formulate placement

problems in terms of grounding in the manner I sketched above, we should

expect new possibilities to open up with respect to how to solve them. I argued

that this is so with respect to the content placement problem in particular. The

grounding view, while quite speculative in nature, deserves further thought.

With recourse to grounding, perhaps naturalizing mental content along

informational lines isn't as hopeless as many think. ${ }^{15}$

\section{References}

Adams, F. and K. Aizawa. 2010. 'Causal Theories of Mental Content', The Stanford Encyclopedia of Philosophy (Spring 2010 Edition), Edward N. Zalta (ed.), $\mathrm{URL}=<\mathrm{http} / /$ plato.stanford.edu/archives/spr2010/entries/content-causal/>.

Audi, P. 2012. 'Grounding: Toward a Theory of the In-Virtue-Of Relation', Journal of Philosophy 109: 685-711.

Aydede, M. and G. Güzeldere. 2005. 'Cognitive Architecture, Concepts, and Introspection: An Information-Theoretic Solution to the Problem of Phenomenal Consciousness', Nous 39: 197-255.

${ }^{15}$ I wish to thank my audiences at the Australian National University, Hong Kong University, Lingnan University, University of Oslo, Shandong University, Shanxi University, and University of Western Australia for their objections and suggestions. Special thanks are due to Ken Aizawa, Louise Antony, Derek Baker, Einar Bohn, Sam Cowling, Chris Daly, Tim Fuller, Michael Johnson, Joe Levine, Jon Litland, Barry Loewer, Dan Marshall, Tristram McPherson, Chris Meacham, Angela Mendelovici, Jennifer Nado, Daniel Nolan, Ted Parent, Adam Pautz, Rob Rupert, Paulo Santorio, Raul Saucedo, Susanna Schellenberg, Wolfgang Schwarz, Michael Strevens, Jonathan Simon, Daniel Stoljar, and Yingjin Xu. 
Bliss, R. and K. Trogdon. 2014. 'Metaphysical Grounding', The Stanford

Encyclopedia of Philosophy (Winter 2014 Edition), Edward N. Zalta (ed.), $\mathrm{URL}=<\mathrm{http}: / /$ plato.stanford.edu/archives/win2014/entries/grounding/>

Bridges, J. 2006. 'Does Informational Semantics Rest on a Mistake?' Nous 40: 522547.

Clark, M. and D. Liggins. 2012. 'Recent Work on Grounding', Analysis 72: 812-823.

Correia, F. and B. Schnieder. 2012. 'Grounding: An Opinioned Introduction', in (ed.)

F. Correia and B. Schnieder, Grounding and Explanation (pp. 1-36).

Cambridge UP.

Dasgupta, S. 2014. 'On the Plurality of Grounds', Philosophers' Imprint 14: 1-28.

Dretske, F. 1995. Naturalizing the Mind. MIT Press.

Fine, K. 1994. 'Essence and Modality', Philosophical Perspectives 8: 1-16.

-2001. 'The Question of Realism', Philosophers' Imprint 1: 1-30.

-2012. 'A Guide to Ground', in (eds) F. Correia and B. Schnieder, Grounding and Explanation (pp. 37-80). Cambridge UP.

Fodor, J. 1990. 'A Theory of Content II: The Theory', in his A Theory of Content and Other Essays (pp. 89-136). MIT Press.

- 1994. The Elm and the Expert: Mentalese and its Semantics. MIT Press.

-1997. 'Special Sciences: Still Autonomous After All These Years', Philosophical Perspectives 11: 149-163.

-1998. Concepts: Where Cognitive Science Went Wrong. Oxford UP.

Gates, G. 1996. ‘The Price of Information', Synthese 107: 325-347.

Jackson, F. 1998. From Metaphysics to Ethics. Oxford UP.

Nolan, D. 2014. 'Hyperintensional Metaphysics', Philosophical Studies 171: 149-160.

Laurence, S. and E. Margolis. 2002. 'Radical Concept Nativism', Cognition 86: 25-55.

Levine, J. 2001. Purple Haze. Oxford UP.

Lewis, D. 1983. 'New Work For a Theory of Universals', Australasian Journal of Philosophy, 61: 343-377. 
Litland, J. 2013. 'On Some Counterexamples to the Transitivity of Grounding', Essays in Philosophy 14/1: 19-32.

Raven, M. 2012. 'In Defense of Ground', Australasian Journal of Philosophy, 90: $687-701$.

— Forthcoming. 'Ground', Philosophy Compass.

Rodriguez-Pereyra, G. Forthcoming. 'Grounding is Not A Strict Order', Journal of the American Philosophical Association.

Rosen, G. 2010. 'Metaphysical Dependence: Grounding and Reduction', in (eds) R. Hale and A. Hoffman, Modality: Metaphysics, Logic, and Epistemology (pp. 109-136). Oxford UP.

Rupert, R. 2008. 'Causal Theories of Mental Content', Philosophy Compass 3: 353380.

Ryder, D. 2009. 'Representation II: Content', in (eds) J. Symons and P. Calvo, The Routledge Companion to Philosophy of Psychology (pp. 251-279). Routledge.

Schaffer, J. 2009. 'On What Grounds What', in (eds) D. Chalmers et al., Metametaphysics (pp. 347-383). Oxford UP.

- 2012. 'Grounding, Transitivity, and Contrastivity', in Correia and Schnieder, pp. $122-138$.

Shapiro, S. 1997. Philosophy of Mathematics: Structure and Ontology. Oxford UP.

Trogdon, K. 2013a. 'An Introduction to Grounding', in (eds) M. Hoeltje, B. Schnieder, and A. Steinberg, Varieties of Dependence. Basic Philosophical Concepts, Philosophia Verlag: 97-122.

-2013b. 'Grounding: Necessary or Contingent?' Pacific Philosophical Quarterly 94: $465-485$. 